\title{
Correspondence
}

\section{Scale issue in fractal analysis of histological specimens}

\section{To the Editor:}

We read with great interest the recent article by Tambasco and Magliocco [1] reporting the application of box counting fractal analysis to the automated measurements of epithelial architectural complexity from histological specimens of breast cancer. While the intention to provide an objective method to quantify architectural complexity may help reducing interobserver discrepancy, we would like to point out that the procedure documented in the paper referred above has a critical pitfall that precludes an objective derivation of the fractal dimension. Specifically, it was stated for their Fig. 6 that the log-log plot, from which the fractal dimension was computed as the slope of the regression line, was "linear over approximately 4 logarithmic scales." A closer examination on the figure, however, suggests that the scale used by Tambasco and Magliocco was actually a natural logarithm, that is, $\ln [N(\varepsilon)]-\ln (1 / \varepsilon)$ rather than $\log _{10}[N(\varepsilon)]-\log _{10}(1 / \varepsilon)$. Otherwise, the number of boxes [ie, $N(\varepsilon)]$ as large as $10^{11}$ would be totally irrational. Therefore, the data shown in Fig. 6 spanned over the range of $e^{(3.5-1)}=12.2$, which was only slightly larger than one order of magnitude. This makes one wonder about the selection of data range for linear regression to obtain the fractal dimension because the box widths were allowed to vary over three orders of magnitude, given that their specimen images were digitized at a $3090 \times 3090$ matrix.

To provide the evidence on the importance of data range selection, we used our histological specimens of renal cell carcinoma obtained from patients undergoing radical nephrectomy, immunohistochemically stained by monoclonal antibodies for the endothelial marker CD34, magnified using an optical microscope at $\times 200$ magnification, digitized with a charge-coupled-device camera mounted to the microscope, and then analyzed in a way similar to that reported by Tambasco and Magliocco [1]. Fig. 1A shows the result of the $\log _{10}[N(\varepsilon)]-\log _{10}(1 / \varepsilon)$ plots for two example specimens and the Koch triadic curve (a synthetic fractal object with known fractal dimension $=1.2628$ ), covering 3 orders of magnitude of box widths. The 2 histological specimens demonstrated similar degrees of variations in the local slopes ranging from 1.8 or 2.0 at large box widths to about 0.9 at small box widths, unlike the Koch triadic curve whose data showed relatively constant slope. When performing linear regression to obtain the slope using box widths spanning only one order of magnitude, the fractal dimension became strongly dependent on the spatial scale included in the analysis (Fig. 1B) [2,3]. Specimen 2 showed stable fractal dimension within about half order of magnitude only, whereas in specimen 1 , no stable fractal dimension could be obtained. The ability to differentiate between these two specimens using fractal dimension also depended on the spatial scale used.

We continued the analysis on a total of 40 different specimens to investigate the dependency of fractal dimension on spatial scale, with fitting range intentionally restricted to be slightly over only 1 order of magnitude. The fractal dimensions derived at different spatial scales were then recorded. The results showed that almost all specimens exhibited fractal dimensions varying from 0.9 to 1.8 as in the case of Fig. 1B, with standard deviation being $0.236 \pm 0.058$ among the 40 samples. In comparison, slope variations for the Koch triadic curve due to spatial scale variation, as demonstrated in Fig. 1B was only 0.034 . Given that the differentiation between tumor grades of the breast histological specimens reported by Tambasco and Magliocco relied on the small difference of about 0.1 (Fig. 8 in Ref. [1]), the variations of $0.236 \pm$ 0.058 in fractal dimension due to spatial scale alone simply cannot be neglected.

Biologic samples, unlike synthetic fractal objects such as the Koch triadic curve, often exhibit self-similarity over an extremely limited range of spatial scale. Whether the limited "fractal property" may invalidate the use of common fractal analysis as a means to quantify structural complexity is still under continuous debate [4,5]. Nevertheless, there is no question that the variations in regression slope of the $\log _{10}[N(\varepsilon)]-\log _{10}(1 / \varepsilon)$ plot entail a careful and objective selection of the spatial scale of the data to be analyzed, in order for the method to be fully automated and to allow reproducible comparison among different groups [6]. We therefore recommend that the spatial scale relevant to the histological specimens 

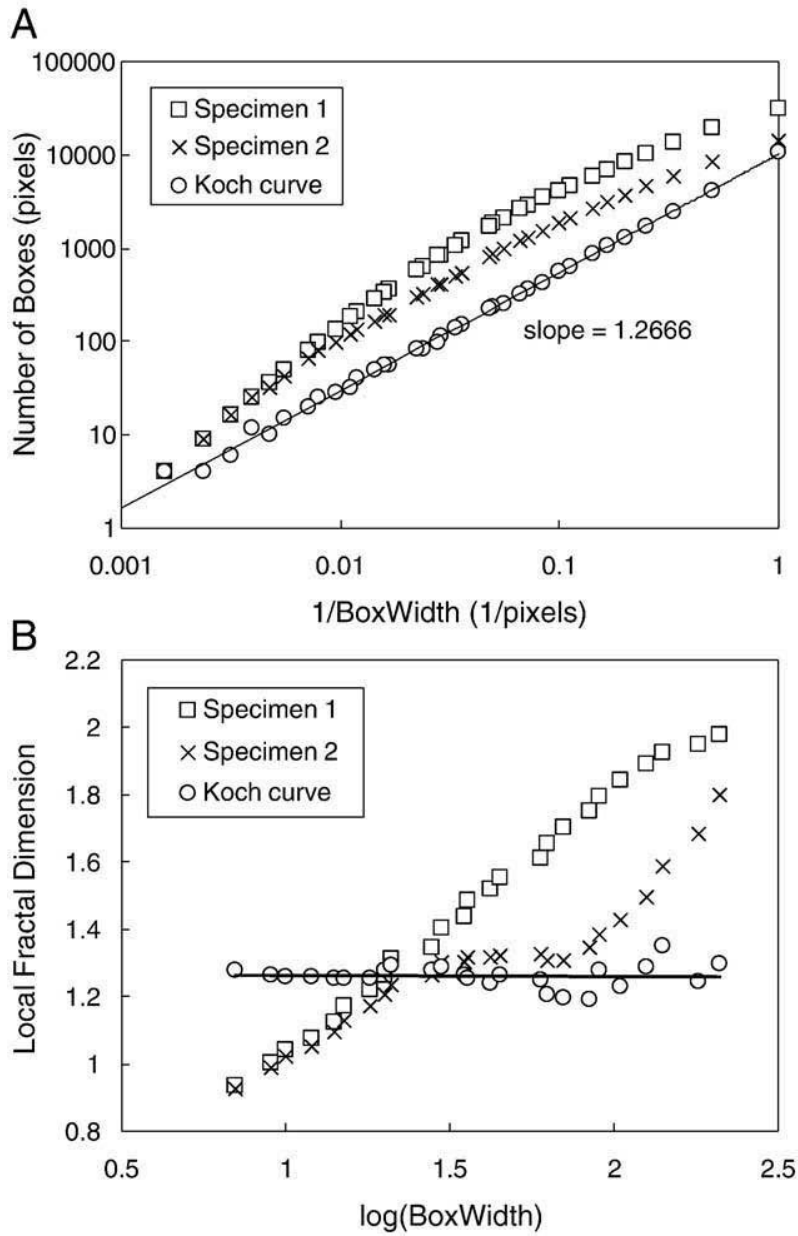

Fig. $1 \mathrm{~A}$, The $\log _{10}[N(\varepsilon)]-\log _{10}(1 / \varepsilon)$ plots for two renal cell carcinoma specimens and the Koch triadic curve, from which the fractal dimensions were derived. In contrast to the Koch triadic curve showing relatively constant slope, the data obtained from the 2 histological specimens showed convex curves. B, Local slopes of the $\log _{10}[N(\varepsilon)]-\log _{10}(1 / \varepsilon)$ plot, obtained via linear regression on data points whose spatial scale was restricted to be within one order of magnitude, showed large variations from about 0.9 at small box widths to 1.8 or 2.0 at large box widths for the 2 specimens. Thus the fractal dimension became strongly dependent on the spatial scale included in the analysis.

under investigation should be explicitly included in reports documenting the use of box counting fractal analysis in biomedical applications.

Hsiao-Jen Chung Division of Urology Department of Surgery Taipei Veterans General Hospital and Department of Urology School of Medicine National Yang-Ming University Taipei, Taiwan, ROC
Hsiao-Wen Chung Department of Electrical Engineering National Taiwan University Taipei 10764, Taiwan, ROC E-mail address: chung@cc.ee.ntu.edu.tw doi:10.1016/j.humpath.2008.09.004

\section{References}

[1] Tambasco M, Magliocco AM. Relationship between tumor grade and computed architectural complexity in breast cancer specimens. Hum Pathol 2008;39:740-6.

[2] Chung HW, Chung HJ. Correspondence re: J. W. Baish and R. K. Jain, Fractals and cancer. Cancer Res., 60: 3683-3688, 2000. Cancer Res 2001;61:8347-50.

[3] Wu WC, Chung HW, Chung HJ. Fractal analysis in liver fibrosis. Hepatology 2003;37:483.

[4] Ciccotti M, Mulargia F. Pernicious effect of physical cutoffs in fractal analysis. Phys Rev E 2002;65:037201.

[5] Avnir D, Biham O, Lidar D, Malcai O. Is the geometry of nature fractal? Science 1998;279:39-40.

[6] Risser L, Plouraboue F, Steyer A, Cloetens P, Le Duc G, Fonta C. From homogeneous to fractal normal and tumorous microvascular networks in the brain. J Cereb Blood Flow Metab 2007;27:293-303.

\section{Scale issue in fractal analysis of histological specimens-reply}

To the Editor:

We thank Chung and Chung for their interest and comments regarding the article we recently published in human pathology. In our article [1], we reported the application of box counting fractal analysis to the automated measurements of epithelial architectural complexity of breast cancer histology sections. In their letter to the editor, Chung and Chung have correctly noted the importance of selecting a suitable data range when performing fractal analysis on biological samples. Chung and Chung also correctly inferred that the scale used in our study was a natural logarithmic scale. To clarify the analysis we performed in our study [1], in this response to Chung and Chung's letter, we provide a more complete description of the method we used to select a suitable data range to compute the fractal dimension from the natural logarithm plots generated by the box counting method.

As mentioned in our article, we applied the box counting method to analyze outline images representing the epithelial structures of breast histology sections [1]. The microscopic images were acquired with an AxioCam HR digital camera (Carl Zeiss Canada Ltd., Toronto, Ontario) mounted on an optical microscope (Zeiss Axioscope) at a magnification of $10 \times$ objective. At a resolution setting of $3090 \times 3900$ pixels (not $3090 \times$ 3090 as incorrectly stated in our article [1]), the pixels 